SHORT REPORT

\title{
High resolution microarray comparative genomic hybridisation analysis using spotted oligonucleotides
}

\author{
B Carvalho, E Ouwerkerk, G A Meijer, B Ylstra
}

J Clin Pathol 2004;57:644-646. doi: 10.1136/icp.2003.013029

Background: Currently, comparative genomic hybridisation array (array $\mathrm{CGH}$ ) is the method of choice for studying genome wide DNA copy number changes. To date, either amplified representations of bacterial artificial chromosomes (BACs)/phage artificial chromosomes (PACs) or cDNAs have been spotted as probes. The production of BAC/PAC and cDNA arrays is time consuming and expensive.

Aim: To evaluate the use of spotted 60 mer oligonucleotides (oligos) for array $\mathrm{CGH}$.

Methods: The hybridisation of tumour cell lines with known chromosomal aberrations on to either BAC or oligoarrrays that are mapped to the human genome.

Results: Oligo $\mathrm{CGH}$ was able to detect amplifications with high accuracy and greater spatial resolution than other currently used array CGH platforms. In addition, single copy number changes could be detected with a resolution comparable to conventional $\mathrm{CGH}$.

Conclusions: Oligos are easy to handle and flexible, because they can be designed for any part of the genome without the need for laborious amplification procedures. The full genome array, containing around 30000 oligos of all genes in the human genome, will represent a big step forward in the analysis of chromosomal copy number changes. Finally, oligoarray CGH can easily be used for any organism with a fully sequenced genome.

A rray comparative genomic hybridisation (array CGH) has been used successfully for the detection of genomic imbalances in human and mouse tumours. ${ }^{1-6}$ As chromosomal representations, approximately 2500 bacterial artificial chromosome (BAC) and phage artificial chromosome (PAC) clones have been amplified and spotted for genome wide CGH arrays, yielding a resolution of $1-1.5 \mathrm{Mb},{ }^{7}$ in addition to cDNAs, ${ }^{8}$ which encompass a maximum of 13824 genes and yield an average resolution of $267 \mathrm{~kb} .{ }^{9}$ Although spatial resolution using cDNAs is currently higher, the number of cDNAs is finite and their sensitivity is lower. This reduced sensitivity of cDNAs is partly the result of cross hybridisation. Oligonucleotides (oligos) can theoretically circumvent the problems encountered with cDNAs. In addition, oligo-libraries are cheaper, easier to work with, and faster than cDNAs or BAC/PAC clones, because no DNA isolation or PCR amplification steps are necessary. The in silico design can control for the hybridisation temperature and specificity and there is no limit to the spatial resolution. Finally, oligos can be designed for any organism with a sequenced genome.

\section{MATERIALS AND METHODS}

BAC array preparation

DNA from the $1 \mathrm{Mb}$ resolution Sanger BAC set (http:// www.ensembl.org/Homo_sapiens/cytoview), the OncoBac set
(http://informa.bio.caltech.edu/Bac onc.html), and in house clones of interest, supplemented with clones from the Children's Hospital Oakland Research Institute (CHORI), amounting to a total of 4202 clones with known chromosomal positions, was isolated according to the CHORI protocol (http://bacpac.chori.org/dnaprep.htm). Amplification of the DNA was done by ligation mediated polymerase chain reaction (PCR) according to Snijders et al, ${ }^{7}$ and PCR clones were purified using Montage $\mathrm{PCR}^{\circledR} \mu 96$ filter plates (Millipore BV, Amsterdam, the Netherlands). Clones were spotted at a concentration of $10 \mu \mathrm{g} / \mu \mathrm{l}$, in $150 \mathrm{mM}$ sodium phosphate $(\mathrm{pH} 8.5)$, on CodeLink ${ }^{\mathrm{TM}}$ slides (Amersham BioSciences, Roosendaal, the Netherlands), using a Spot Array 72 robot (Perkin Elmer Life Sciences, Zaventum, Belgium), and processed according to the manufacturer's protocol.

\section{Oligoarray preparation}

A collection of 18861 oligo sequences, representing 18664 unique genes (Sigma-Genosys, Zwijndrecht, the Netherlands), was spotted at a concentration of $10 \mu \mathrm{M}$, in $150 \mathrm{mM}$ sodium phosphate ( $\mathrm{pH}$ 8.5), on CodeLink ${ }^{\mathrm{TM}}$ slides (Amersham BioSciences), using a Spot Array 72 robot (Perkin Elmer Life Sciences), and processed according to the manufacturer's protocol.

\section{Hybridisation procedure}

Genomic DNA was isolated from normal kidney (female donor), blood (male donor), or liver (female donor) as a reference, and from the breast cancer cell line BT474, according to Snijders et al. ${ }^{7}$ The DNA from the GM00143 cell line (Coriell Institute for Medical Research, Camden, New Jersey, USA) was provided by the Albertson laboratory (University of California, San Francisco, USA). For the BAC arrays, DNA labelling and hybridisation were performed according to Snijders et al, ${ }^{7}$ using ProbeQuant ${ }^{\mathrm{TM}}$ G50 microcolumns (Amersham Biosciences). For the oligoarray hybridisations, the concentration of formamide was decreased to $34 \%$ and washes were performed at $36^{\circ} \mathrm{C}$ to compensate for the reduced target length. Before scanning slides (Scan Array Express; Perkin Elmer Life Sciences) both oligoarrays and BAC arrays were dried by centrifugation $(141 \times g)$, omitting DAPI staining. Spots were quantified by Imagene 5.5.4 software (Biodiscovery Ltd, Marina del Rey, California, USA), using the default settings. Subtraction of local background was done for the signal mean intensities in both test and reference DNA channels. Normalisation in the calculated ratios was done against the average of all ratios. On the BAC arrays, because the clones were spotted in triplicate, average and standard deviations were calculated

Abbreviations: $\mathrm{BAC}$, bacterial artificial chromosome; $\mathrm{CGH}$, comparative genomic hybridisation; CHORI, Children's Hospital Oakland Research Institute; oligo, oligonucleotide; PAC, phage artificial chromosome; PCR, polymerase chain reaction 

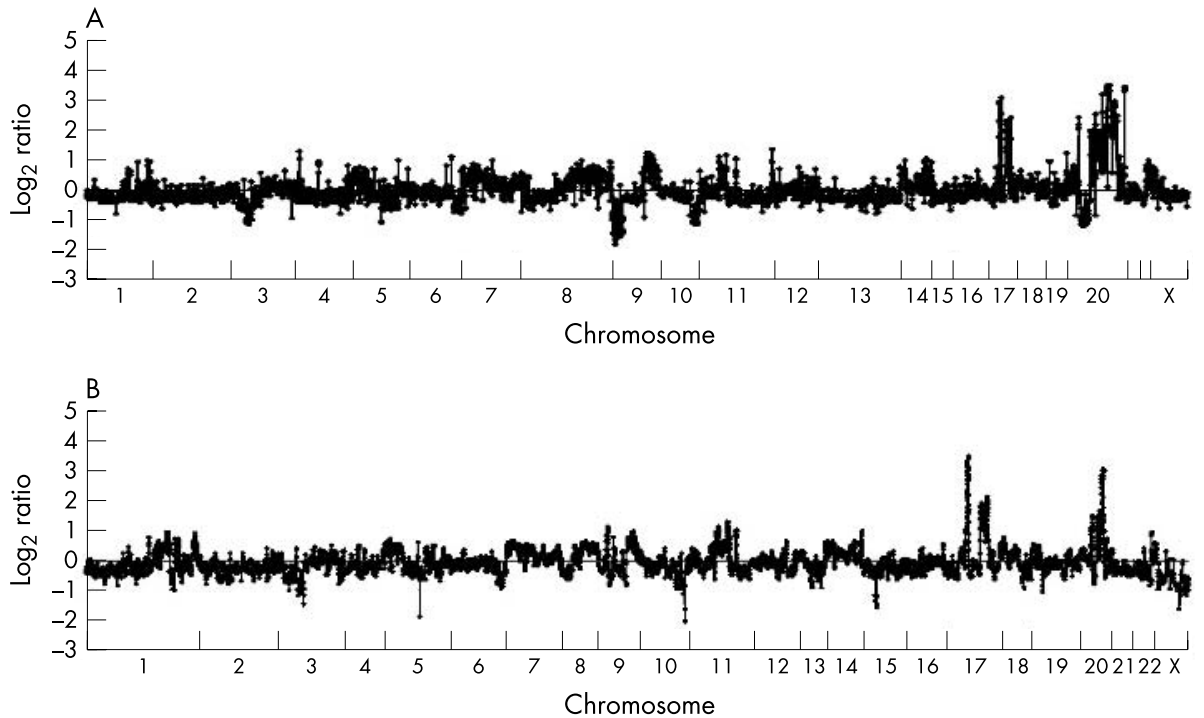

Figure 1 Microarray comparative genomic hybridisation (CGH) profile of the BT474 cell line. (A) Bacterial/phage artificial chromosome polymerase chain reaction representations were used as probe DNAs spotted on to the glass slide; (B) oligonucleotides were used as probe DNAs spotted on to the glass slide. Moving average applied to the $\log _{2}$ ratio of the oligo CGH profile. The vertical bars indicate the spacing between the chromosomes. for each clone. On the oligoarrays, each experiment was performed three times and data were taken from one representative experiment. Average and standard deviations of $\log _{2}$ ratios were calculated for each oligonucleotide across the three experiments. A moving average (window of eight by eight) was applied to plot genome wide graphs.

\section{RESULTS AND DISCUSSION}

We hybridised a $19 \mathrm{~K}$ human 60 mer oligoarray with breast tumour cell line (BT474) DNA, labelled with Cy3, and normal genomic kidney (female) DNA, labelled with Cy5. Ratios for the non-flagged oligos (35\%) were ordered by their position on the chromosome (June 2002 freeze; http://genome.ucsc. edu/). We compared the oligo CGH profile with the BAC array CGH profile (fig 1). Both array profiles showed the same pattern-for example, on the short arm of chromosome 1 neither profile showed a change in DNA copy number, whereas on the q arm two amplified areas are present in both profiles. No aberrations can be seen on chromosome 2 . The standard deviation of the $\log _{2}$ ratio of the individual probes was 0.21 for the BAC array and 0.45 for the oligoarray. On chromosome 3, a loss on the short arm was evident on the oligoarray, and was also seen with the BAC array (fig 1). Figure 2 shows two regions of amplification on the $\mathrm{q}$ arm of chromosome 17: one narrow peak over the chromosomal region containing c-Erb-B2/neu (Her2 $)^{10}$ and a second amplicon distal to c-Erb-b2. The BAC array has three clones over C-Erb-B2, and the best possible judgment towards the start and end of the amplicon, according to the April 2003 freeze, is therefore $2.5 \mathrm{Mb}$. With the oligo approach, 38 non-flagged oligos represent amplified ratios in this region and the size of the amplicon is $2.4 \mathrm{Mb}$ according to the April 2003 freeze. Thus, the actual resolution in the region is $63 \mathrm{~kb}$ on average. The $\log _{2}$ ratios for the three replicate BACs containing the c-Erb-b2 gene are 2.91, 3.06, and 2.53, a similar order of magnitude as that obtained in three independent experiments with the oligoarray for the single oligo corresponding to the c-Erb-b2 gene: 3.4, 3.4, and 3.9.
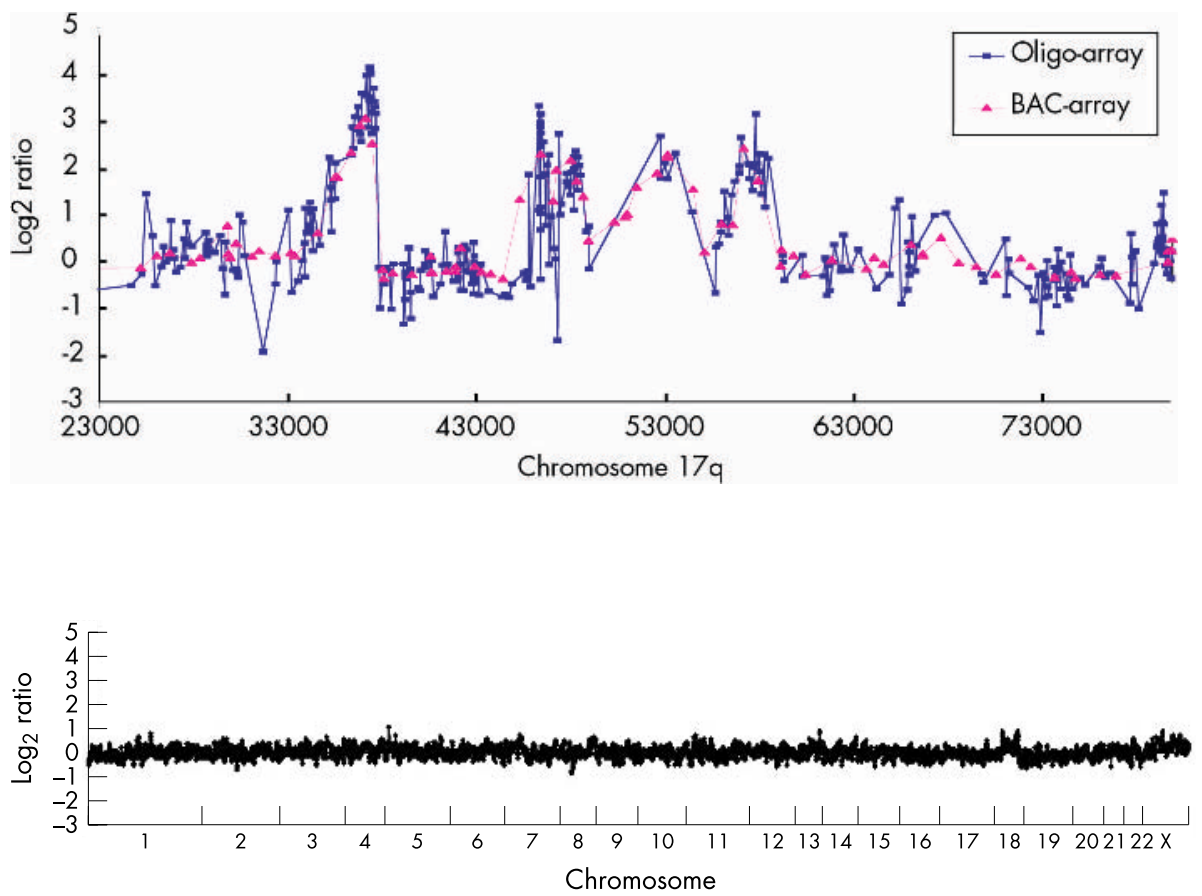

Figure 2 Microarray comparative genomic hybridisation (CGH) profile of the long arm of chromosome 17 of the BT474 cell line. Grey triangles, bacterial/phage artificial chromosome polymerase chain reaction representations were used as probe DNAs spotted on to the glass slide; black squares, oligonucleotides were used as probe DNAs spotted on to the glass slide $\left(\log _{2}\right.$ ratio without moving average). The horizontal bars indicate the two separate amplicons observed.

Figure 3 Microarray comparative genomic hybridisation profile of the GM00143 cell line with a trisomy of chromosome 18. Moving average applied to the $\log _{2}$ ratio. The vertical bars indicate spacing between the chromosomes. 


\section{Take home message}

- We describe pilot experiments that serve as a proof of principle that oligonucleotides are a feasible platform for array comparative genomic hybridisation (CGH)

- Oligoarray CGH can be rapidly, cost effectively, and easily used to measure chromosomal copy number changes for any organism with a fully sequenced genome

The same observation can be made for the second amplicon on chromosome 17q.

The numbers for the comparison of those specific arrays not withstanding, it is obvious that an almost infinite amount of oligos can be rapidly synthesised for a specific region of interest, whereas the BACs are limited, especially by size, but also because availability and processing of the BACs is slower than for oligos.

As a further test of whether the oligo CGH is sensitive enough to detect single copy number changes, we performed a hybridisation with DNA from cell line GM0143 (female donor), which has trisomy of chromosome 18, and normal liver DNA (female donor). The theoretical ratio of a trisomy should be $1.5\left(\log _{2}\right.$ ratio, 0.58$)$ and the BAC array showed a $\log _{2}$ ratio value of $0.49(\mathrm{SD}, 0.05) .^{7}$ For the oligoarray the average of the $\log _{2}$ ratio was 0.31 (SD, 0.49) (fig 3). Although this single gain can be clearly distinguished by using a moving average (eight by eight), the high standard deviation indicates the need to optimise the hybridisation conditions.

"An almost infinite amount of oligos can be rapidly synthesised for a specific region of interest, whereas the bacterial artificial chromosomes are limited, especially by size"

In this first set of experiments, the percentage of spots that were flagged was very high $(65 \%)$. These non-hybridising oligos could be partially explained by the fact that some oligos span exon-exon boundaries. Therefore, a dedicated oligo library specifically designed for array CGH, analogous to the situation with expression microarrays, could improve the performance of oligo CGH even further.

\section{ACKNOWLEDGEMENTS}

We are indebted to R M Reis, P P Eijk, M Tijssen, and P van den IJssel for printing slides and help with hybridisation protocols. We would like to thank Professor D G Albertson, Professor D Pinkel, and laboratory staff (UCSF Comprehensive Cancer Centre) for their support in performing the hybridisation procedures and for the GM0143 DNA sample. BC is holder of fellowship SFRH/BPD/5599/ 2001 and is working in the frame of the Grant Project POCTI/CBO/ $41179 / 2001$. This work is furthermore supported by the Dutch Cancer Society (VU 2002-2618). We thank the mapping core and map finishing groups of the Wellcome Trust Sanger Institute for initial BAC clone supply and verification.

\section{Authors' affiliations}

B Carvalho, G A Meijer, Department of Pathology, VU University Medical Centre, de Boelelaan 1117, 1081HV Amsterdam, The Netherlands

E Ouwerkerk, B Ylstra, Microarray Core Facility, Medical Faculty, VU University Medical Centre, van der Boechorststraat 7-9, 1081BT

Amsterdam, The Netherlands

Correspondence to: $\operatorname{Dr} B$ Ylstra, Microarray Core Facility, Medical Faculty, VU University Medical Centre, van der Boechorststraat 7-9, 1081BT Amsterdam, The Netherlands; b.ylstra@vumc.nl

Accepted for publication 13 January 2004

\section{REFERENCES}

1 Albertson DG, Ylstra B, Segraves R, et al. Quantitative mapping of amplicon structure by array CGH identifies CYP24 as a candidate oncogene. Nat Genet 2000;25:144-6.

2 Hodgson G, Hager JH, Volik S, et al. Genome scanning with array CGH delineates regional alterations in mouse islet carcinomas. Nat Genet 2001;29:459-64.

3 Pollack JR, Sorlie T, Perou CM, et al. Microarray analysis reveals a major direct role of DNA copy number alteration in the transcriptional program of human breast tumours. Proc Natl Acad Sci U S A 2002;99:12963-8.

4 Wilhelm M, Veltman JA, Olshen AB, et al. Array-based comparative genomic hybridization for the differential diagnosis of renal cell cancer. Cancer Res 2002;62:957-60.

5 Snijders AM, Nowee ME, Fridlyand J, et al. Genome-wide-array-based comparative genomic hybridization reveals genetic homogeneity and frequent copy number increases encompassing CCNE 1 in fallopian tube carcinoma. Oncogene 2003;22:4281-6.

6 Weiss MM, Kuipers EJ, Postma C, et al. Genomic profiling of gastric cancer predicts lymph node status and survival. Oncogene 2003;22: 1872-9.

7 Snijders AM, Nowak N, Segraves R, et al. Assembly of microarrays for genome-wide measurement of DNA copy number. Nat Genet 2001;29:263-4.

8 Pollack JR, Perou CM, Alizadeh AA, et al. Genome-wide analysis of DNA copy-number changes using cDNA microarrays. Nat Genet 1999;23:41-6.

9 Hyman E, Kauraniemi P, Hautaniemi S, et al. Impact of DNA amplification on gene expression patterns in breast cancer. Cancer Res 2002;62:6240-5.

10 Coussens L, Yang-Feng TL, Liao Y-C, et al. Tyrosine kinase receptor with extensive homology to EGF receptor shares chromosomal location with NEU oncogene. Science 1985;230:1132-9. 